\title{
Are tachoclines important for solar and stellar dynamos? What can we learn from global simulations
}

\author{
G. Guerrero ${ }^{1}$, P. K. Smolarkiewicz ${ }^{2}$, E. M. de Gouveia Dal Pino ${ }^{3}$, \\ A. G. Kosovichev ${ }^{4}$, B. Zaire ${ }^{1}$ and N. N. Mansour ${ }^{5}$ \\ ${ }^{1}$ Physics Department, Universidade Federal de Minas Gerais, \\ Av. Presidente Antonio Carlos 6627, Belo Horizonte, MG, 31270-901, Brazil, \\ email: guerrero@fisica.ufmg.br \\ ${ }^{2}$ European Centre for Medium-Range Weather Forecasts, Reading RG2 9AX, UK \\ ${ }^{3}$ Astronomy Department, IAG-USP, Rua do Matão, 1226, SP, 05508-090, Brazil \\ ${ }^{4}$ New Jersey Institute of Technology, Newark, NJ 07103, USA \\ ${ }^{5}$ NASA, Ames Research Center, Moffett Field, Mountain View, CA 94040, USA
}

\begin{abstract}
The role of tachoclines, the thin shear layers that separate solid body from differential rotation in the interior of late-type stars, in stellar dynamos is still controversial. In this work we discuss their relevance in view of recent results from global dynamo simulations performed with the EULAG-MHD code. The models have solar-like stratification and different rotation rates (i.e., different Rossby number). Three arguments supporting the key role of tachoclines are presented: the solar dynamo cycle period, the origin of torsional oscillations and the scaling law of stellar magnetic fields as function of the Rossby number. This scaling shows a regime where the field strength increases with the rotation and a saturated regime for fast rotating stars. These properties are better reproduced by models that consider the convection zone and a fraction of the radiative core, naturally developing a tachocline, than by those that consider only the convection zone.
\end{abstract}

Keywords. Stars; rotation; Stars: magnetism; Stars: dynamo

\section{Introduction}

In stellar interiors, tachoclines are interface layers where the solid body rotation of the stars interior becomes differential rotation. It is also associated with the mechanism by which the star transfers energy from the core to the photosphere, i.e., the interface between a radiative and a convective layers where rotating turbulence drives the transport of energy and angular momentum. Thus, the location of the tachocline depends on the evolutionary stage of the star. In the case of the Sun it is located at about $71 \%$ of the solar radius.

Since strong gradients of angular velocity occur at these layers, it is believed that they are relevant for the stellar dynamos. For the solar case, particularly, some dynamo models (Dikpati \& Charbonneau 1999; Chatterjee et al. 2004; Guerrero \& de Gouveia Dal Pino 2008) assume that the large-scale toroidal magnetic field generated at the tachocline directly manifests as sunspots, the regions of strong radial magnetic field observed at the surface. A buoyancy process is invoked as the transport agent of magnetic flux ropes. This scenario of solar dynamo is controversial and matter of debate in the literature Guerrero \& Käpylä (2011). However, the same mechanism has already been considered for stars with deeper convection zones.

As for solar-type stars in different evolutive stages, observations of X-ray luminosities, a proxy of stellar activity (Pizzolato et al. 2003; Wright et al. 2011), or magnetic 
fields (and references therein Vidotto et al. 2014), indicate two regimes of activity as function of the Rossby number, Ro. For higher values of the Rossby number (buoyancy dominated convection) the stellar activity follows a exponential relation $\left(L x \propto \mathrm{Ro}^{-2.7}\right.$, $\langle B\rangle \propto \mathrm{Ro}^{-1.39}$ ). For low Rossby numbers (rotation dominated convection) the stellar activity shows a saturated regime. In the sample studied by Wright et al. (2011), the stars in the saturated regime are either partly or fully convective, whereas in the buoyancy dominated regime the stars have already developed a radiative core. This suggested a relation between photospheric and coronal magnetic field and the field developed at the tachocline. Most recently, however, Wright \& Drake (2016) reported new observations of fully convective stars that fall within the rotation dominated regime and follow the $L x \propto \mathrm{Ro}^{-2.7}$ scaling. This result questions the relevance of the tachocline in the generation of the large-scale magnetic field observed in stars. It suggest that in partly convective stars the field does not develop in the shear region but in the convection zone. A distributed dynamo process, with a turbulent $\alpha$-effect (Parker 1955; Steenbeck et al. 1966), is then invoked as the field generating mechanism. The field observed in the Sun, in the form of sunspots, and in other solar-type stars, via Zeeman-Doppler Imaging (Donati \& Brown 1997; Petit et al. 2008), might be of shallow origin as proposed one decade ago by Brandenburg (2005).

To understand the physics behind the stellar dynamo mechanism in a self-consistent form nowadays we draw upon global MHD models. Although these simulations are still far from the numerical resolution required to capture the full dynamics of the stellar interior, they have been successful in reproducing some key ingredients of the dynamo. For instance Browning (2008) found dynamo action in fully convective models mimicking M dwarfs while Brown et al. (2008) obtained steady magnetic field solutions, in simulations of rapidly rotating convection with solar-like stratification. More recently Ghizaru et al. (2010) reported oscillatory dynamo solutions in ILES simulations of convection rotating at the solar rate. Thereafter different groups have found dynamos with periodic magnetic fields in simulations with different Rossby numbers (Käpylä et al. 2012; Guerrero et al. 2016a; Augustson et al. 2015). From these models, the natural development of a tachocline is reproduced only by Ghizaru et al. (2010) and (Guerrero et al. 2016a) who used the EULAG-MHD code. In fact, Guerrero et al. (2016a) presented a comparison between models with and without a stable layer at the bottom of the domain. Their results remark important differences between both kind of models performed with the same code, as well as with models performed with different codes that only consider the convection zone.

In this work we present what we consider the most relevant features observed in simulations of solar-type stars that include a fraction of the radiative zone (and therefore a tachocline). The first two sections extrapolate the results of the models to the solar dynamo case. They discuss how MHD instabilities at the tachocline might set the dynamo period, and how the so-called torsional oscillations might develop in the same region before propagating upwards up to the solar surface. The third section discusses the scaling of the dynamo generated magnetic field with the Rossby number. We present the results of a large number of simulations with (RC models) and without tachoclines (CZ models) which indicate that the models that consider the stable radiative zone are the ones that better reproduce the observational laws discussed above.

\section{The solar dynamo period}

One of the most relevant quantities defining the evolution of a dynamo generated magnetic field is the turbulent magnetic diffusivity, $\eta_{t}$. Given the values for temperature and density in the convection zone, the molecular, Ohmic, magnetic diffusivity is small 

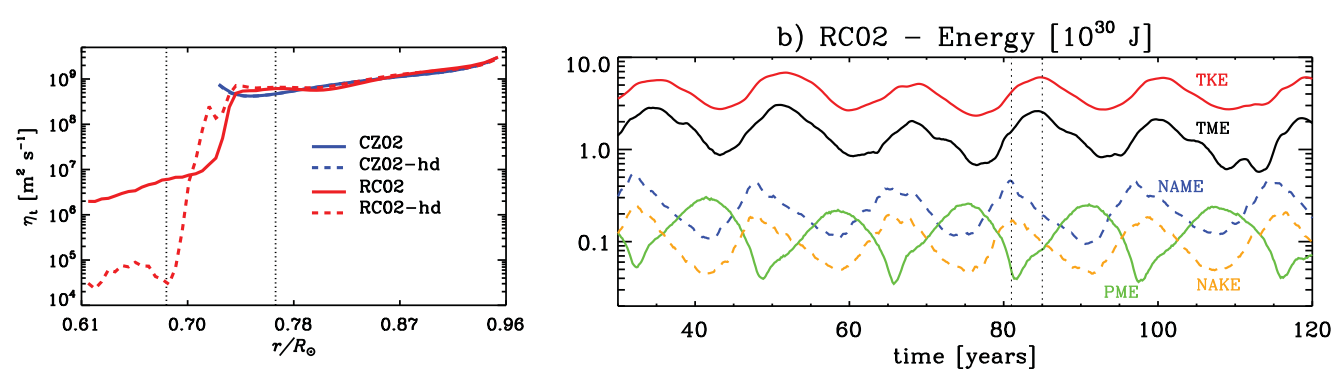

Figure 1. Left: Turbulent magnetic diffusivity of models without (blue) and with radiative zone (red). Solid an dashed lines correspond to MHD and HD cases, respectively. Rigth: Volume integrated energies of the different quantities in the simulation; TKE, TME and PME stand for toroidal kinetic and magnetic energies, and for the poloidal magnetic energy. NAME and NAKE correspond to the non-axisymmetric magnetic and kinetic energies, respectively. Adapted from Guerrero et al. (2016a).

enough $\left(\sim 10^{4} \mathrm{~m}^{2} \mathrm{~s}^{-1}\right)$ to allow a oscillatory dynamo with $22 \mathrm{yr}$ period. According to dynamo theory the rate at which magnetic field is diffusing is competing with the amount of magnetic field being generated and advected. This competition defines what parameter influences more in setting the oscillation period. The value of $\eta_{\mathrm{t}}$ has been estimated from the mixing length theory (e.g., Muñoz-Jaramillo et al. 2011) and also from the properties of the observed butterfly diagram (Cameron \& Schüssler 2016). Both estimations agree with values between $10^{8}-10^{9} \mathrm{~m}^{2} \mathrm{~s}^{-1}$. If this is the case the magnetic diffusivity must be controlling the cycle period of the solar dynamo. Turbulence diffusivity quenching, due to the non-linear interaction between the large scale magnetic field and the turbulent flows, has proven to be inefficient for the period to be controlled by, for example, the meridional circulation (Guerrero et al. 2009; Muñoz-Jaramillo et al. 2011).

What is puzzling about this estimative for the value of $\eta_{\mathrm{t}}$ is that it will diffuse the magnetic field in the convection zone in about 1-2 years, but not in 11 . The simulation without tachocline, CZ02, presented in Guerrero et al. (2016a) results in a value of the turbulent diffusivity, $\eta_{\mathrm{t}}=1.09 \times 10^{9} \mathrm{~m}^{2} \mathrm{~s}^{-1}$ (see blue line in Fig. 1(left)) and a full-cycle period of $2.21 \mathrm{yr}$. The model, including radiative zone, $\mathrm{RC} 02$, results in $\eta_{\mathrm{t}}=1.12 \times 10^{9}$ $\mathrm{m}^{2} \mathrm{~s}^{-1}$ (see red line in 1 (left)) and a cycle period of full-cycle period of $34.5 \mathrm{yr}$. By comparing the solid (MHD case) and the dashed (HD case) red lines in Fig. 1(left), it is possible to notice that $\eta_{\mathrm{t}}$ is not quenched by the large-scale magnetic field in the convection zone. However, it is quenched in the range $0.7 R_{\odot} \lesssim r \lesssim 0.73 R_{\odot}$, where the stronger magnetic field is generated. Interestingly, the evolution of the magnetic field in the rest of the computational domain seems to be governed by the deep seated magnetic field.

According to the results presented in Guerrero et al. (2016a), the cycle period is set by the value of the turbulent diffusivity at the bottom of the convection zone and the radiative layer, $\eta_{\mathrm{t}} \simeq 5 \times 10^{6} \mathrm{~m}^{2} \mathrm{~s}^{-1}$. This value is the result of turbulent motions that develop at the tachocline and the stable layer due to current-driven instabilities (e.g., Cally et al. 2003; Miesch et al. 2007). Because of the shear profile and the configuration of toroidal magnetic field an instability develops. It grows, exchanging energy with the magnetic field (see black and green solid lines in 1(right)). When the magnetic field is weak, the energy of the turbulent motions and magnetic field decays (see dashed lines in Fig. 1(right)). Thus, both the shear and the magnetic field adjust themselves to a equilibrium. Similar results have been obtained for a different global simulation performed with the EULAG-MHD code (Lawson et al. 2015). 


\section{Torsional oscillations}

The slow-down and speed-up of the angular velocity observed at the solar surface are called torsional oscillations (TO). When their latitudinal distribution is plotted against time, two branches can be observed. One of them, with the largest amplitude, migrates towards the poles, the second one migrates equatorwards. Since these branches oscillate with the same periodicity than sunspots, it is believed that the TO are correlated with the large-scale magnetic field. In fact, some correlation is observed between the equatorial branch of TO and the sunspot latitudes of activity. However, the speed-up of the angular velocity starts few years before than the sunspot cycle.

The model RC02 of Guerrero et al. (2016a) shows variations of the angular velocity that resemble quite well the TO pattern, i.e, there are two brances, one polar and one quatorial, that oscillate with the dynamo period (see the upper panel of Fig. 2(a)). The origin of these oscillations was studied in Guerrero et al. (2016b). It was found that the pattern observed at the surface of the model $\left(r=0.95 R_{\odot}\right)$ does not form locally but it is the result of the strong axial torque carried on by the large-scale magnetic tension at the tachocline. Therefore, the morphology of the TO does not corresponds to the magnetic field itself, but to the divergence of the correlations $\bar{B}_{\phi} \bar{B}_{r}$ and $\bar{B}_{\phi} \bar{B}_{\theta}$ at the bottom of the convection zone. This perturbation propagates upwards as can be seen in Fig. 3 where the time evolution of the axial torques is compared with the angular velocity perturbation, $\delta \Omega(r, \theta, t)=(2 \pi \varpi)^{-1}\left(u_{\phi}(r, \theta, t)-\bar{u}_{\phi}(r, \theta)\right)$, where $\varpi=r \sin \theta$ and $(2 \pi \varpi)^{-1} \bar{u}_{\phi}$ is the zonal and temporal average of the angular velocity. In this figure the computational domain has been divided in 6 regions over which volume averages of the quantities are performed. Regions R31, R21 and R11 correspond to the polar, middle latitudes and equatorial tachocline; R32, R22 and R12 to the same latitudes and the bulk of the convection zone; and R33, R23 and R13 to the top of the domain. It can be seen that in the tachocline region, the shape of the TO closely resembles that of the magnetic tension torque with a slight delay. This resemblance disappears in the other regions but the shape of the TO propagates upwards while loosing some amplitude.

From this study, Guerrero et al. (2016b) found that, in general, the meridional circulation appears as a large-scale motion that compensates for the axial torque due to the Reynolds stresses. This is the so-called gyroscopic pumping mechanism (e.g., Miesch \& Hindman 2011) for a quase-steady system (i.e., while full stationarity is never obtained due to the cyclic magnetic field, the establishment of the meridional circulation occurs in a faster time-scale). The steady profile of the meridional circulation varies in a time-scale compared with the magnetic cycle. While at the tachocline, this change is the response to the net magnetic tension torque, at the surface, where the magnetic tension is weaker, the variation is a local response to the TO. This can be seen in panel (b) of 2 where $\delta u_{\theta}=u_{\theta}(r, \theta, t)-\bar{u}_{\theta}(r, \theta)$ is presented in time-latitude (at $r=0.95 R_{\odot}$, upper panel) and time-radius (at $30^{\circ}$ latitude, bottom) butterfly diagrams are presented. The correlation between $\delta u_{\phi}$ and $\delta u_{\theta}$ is evident at the surface with a residual poleward (equatorward) flow appearing when the rotation speeds-up (slows-down). The bottom panel of the same figure indicates that this correlation does not exist all depths, as expected. The correlation between the TO (black line) and a negative axial torque due to the meridional circulation (red line) can be seen in the top and bottom rightmost panels of Fig. 3. It is worth noticing that a similar correlation between $\delta u_{\phi}$ and $\delta u_{\theta}$ has been reported by Komm et al. (2015). These result ultimately indicates that the meridional circulation must be correlated with the magnetic field at the bottom of the convection zone. 

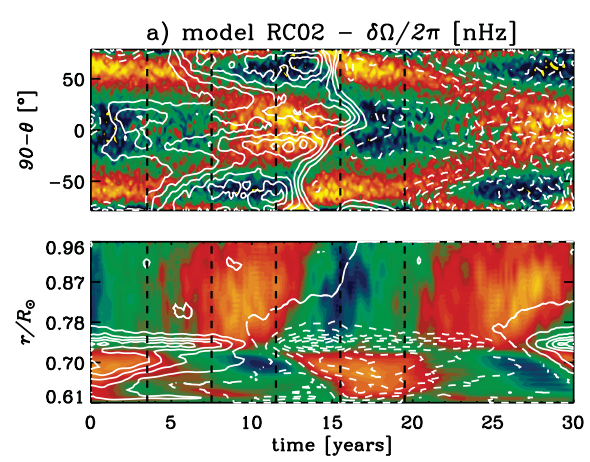
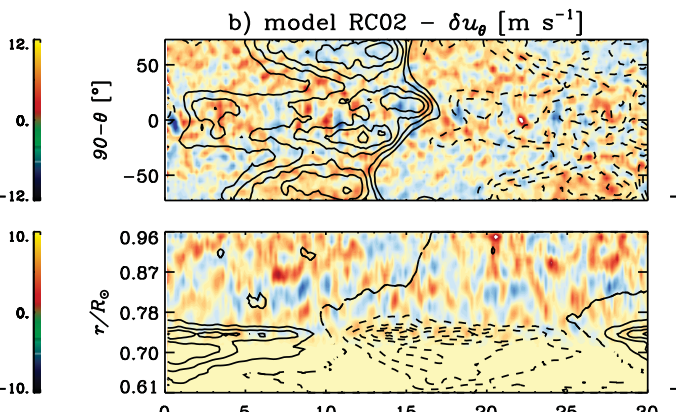

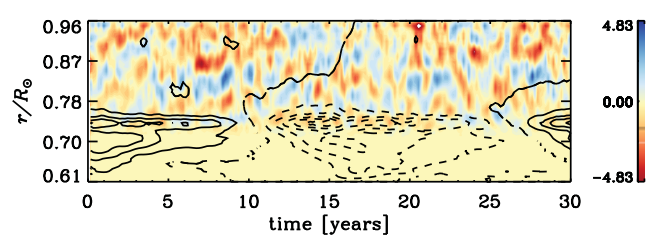

Figure 2. Time-latitude diagram at $r=0.95 R_{\odot}$ (upper panel), and time-radius diagram at $30^{\circ}$ latitude (bottom panel) of $\delta \Omega(r, \theta, t) / 2 \pi=(2 \pi \varpi)^{-1}\left(u_{\phi}(r, \theta, t)-\bar{u}_{\phi}(r, \theta)\right)$, left, and $\delta u_{\theta}=u_{\theta}(r, \theta, t)-\bar{u}_{\theta}(r, \theta)$, right. The continuous (dashed) line contours depict the positive (negative) toroidal magnetic field shown in Fig. 6 of Guerrero et al. (2016a).

(a) R31

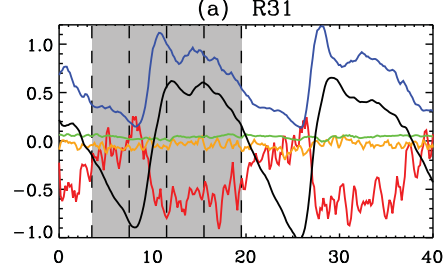

(d) R21

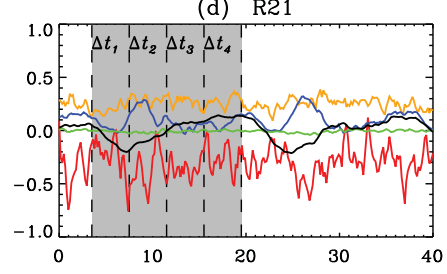

(g) R11

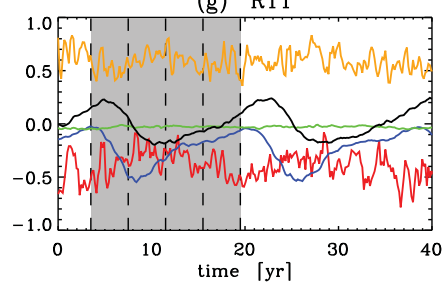

(b) R32

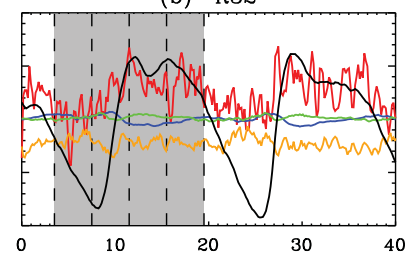

(e) R22

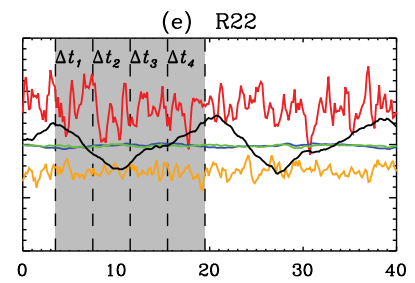

(h) R12

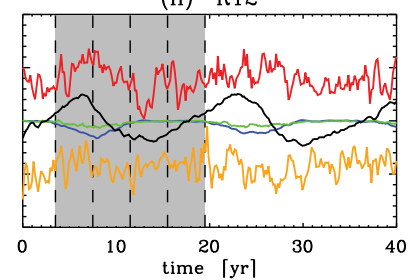

(c) R33
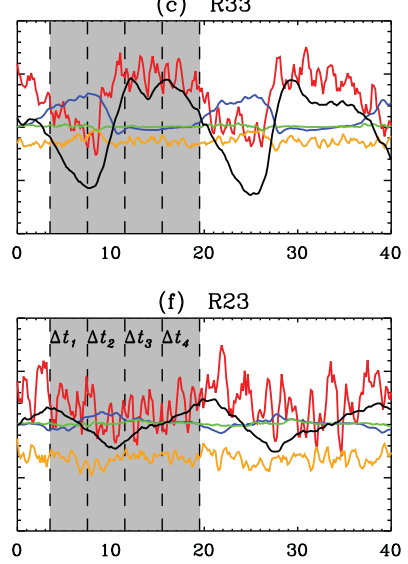

(i) R13

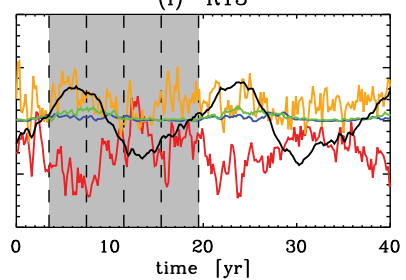

Figure 3. Time evolution of the axial torques computed for different latitudinal and radial regions (see the text). Red, orange, blue and green lines correspond to the meridional circulation (MC), Reynolds stresses (RS), magnetic tension (MT) and Maxwell stresses (MS) axial torques normalized to the local maximum value of $\left\langle\nabla \cdot \mathcal{F}_{\mathrm{MC}}\right\rangle$. The black line shows the evolution of $\langle\delta \Omega\rangle$ normalized to $10^{-7}$. The angular brackets mean volume averages over each region. Adapted from Guerrero et al. (2016b).

\section{Saturation of magnetic field in solar-type stars}

In this section we present results from dynamo simulations of solar-type stars with different Rossby numbers. From these models only six cases were studied in detail in Guerrero et al. (2016a). Here we present eight simulations which include a fraction of the radiative zone ( $\mathrm{RC}$ cases) and seven cases which consider only the convection zone (CZ cases). In Fig. 4 we present the amplitude of the magnetic field as a function of Ro. The 

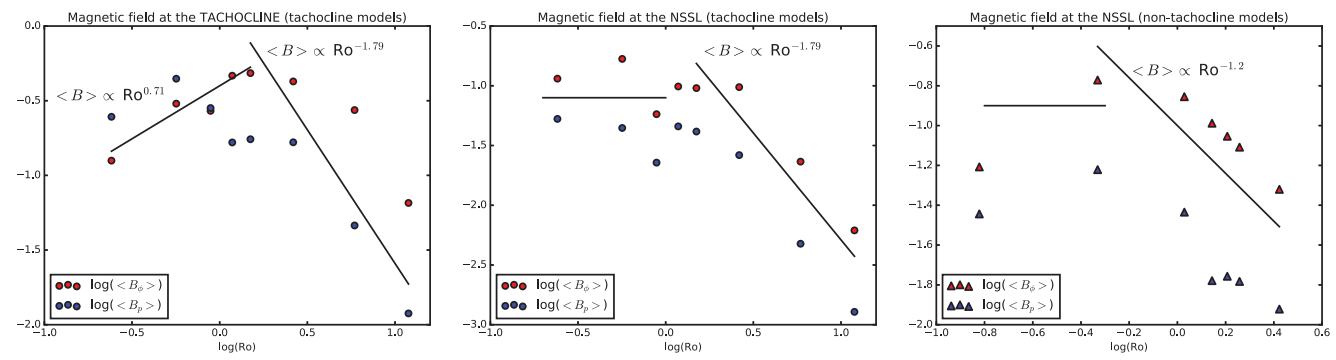

Figure 4. Magnetic field strength as a function of Ro for global simulations that include the radiative zone (left and middle panels) and for models that consider the convection zone only (right). In the left panel the field is measured at the shear region, $0.72 R_{\odot} \leqslant r \leqslant 0.75 R_{\odot}$. In the middle and right panels the field is measured at surface levels, $0.92 R_{\odot} \leqslant r \leqslant 0.96 R_{\odot}$. Red and blue symbols correspond to toroidal and poloidal field components, respectively.

left and middle panels corresponds to RC models while the right panel shows the results for $\mathrm{CZ}$ models. In left panel the rms field is computed in the tachocline region where the shear is stronger $\left(0.72 R_{\odot} \leqslant r \leqslant 0.75 R_{\odot}\right)$. In the middle panel the field is computed in the near-surface shear layer (NSSL, $0.92 R_{\odot} \leqslant r \leqslant 0.96 R_{\odot}$ ). The Rossby number has been computed here by using the definition used by Landin et al. (2010): Ro $=P_{\text {rot }} / \tau$, where $P_{\text {rot }}$ is the rotation period and $\tau=H_{p} / u_{\text {rms }}$ is the convective turnover time computed one pressure scale height above the tachocline.

Starting from the slow rotation regime, at tachocline levels, the toroidal magnetic field (red dots) increases with the decreasing of the Rossby number until Ro $\gtrsim 1$, for lower values of Ro, it exhibits a decay. This happens because faster rotating models result in less shear at the tachocline. The latitudinal shear also decreases and the rotations turns more and more homogeneous. The continuous lines show scaling laws for the magnetic field varying as $\mathrm{Ro}^{0.71}$ and $\mathrm{Ro}^{-1.79}$ for lower and larger values of the Rossby number, respectively. The amplitude of the poloidal magnetic field (blue dots) also increases with the decreasing of the Ro for slow rotating models. For $1 \lesssim$ Ro $\lesssim 3$, the $\left\langle B_{p}\right\rangle$ shows a platoo, afterwards it increases and then decays again.

The scaling is different when the rms magnetic field is computed at the NSSL levels. Both, the toroidal and the poloidal components increase with the decrease of Ro for slow rotating models, but then they reach a saturation level. It seems, however, that the Rossby number of saturation is different for $\left\langle B_{\phi}\right\rangle$ (Ro $\left.\sim 2.5\right)$ than for $\left\langle B_{p}\right\rangle$ (Ro $\left.\sim 1\right)$. The continuous line shows a scaling law with $\mathrm{Ro}^{-1.79}$. Although the shear is decreasing for fast rotating models, the amplitude of the toroidal field remains constant. It could be argued that the $\alpha$ effect increases with the rotation so that $\alpha^{2}$ dynamos are operating in these cases. However, the same analysis for models without the stable layer (right panel of Fig. 4) indicate that this might not be the case. For these models the rms large-scale magnetic field is computed only at the NSSL because there is not a radial shear layer at the bottom of the domain. From right to left, the magnetic field increases for decreasing Ro (the scaling law in this case fits better for $\mathrm{Ro}^{-1.2}$ ) but then decreases quickly for the faster rotating models. Similar behaviour is observed for both, toroidal and poloidal components of the large-scale magnetic field.

This result indicates that the presence of the tachocline is relevant for reproducing the observed saturation of the magnetic field mentioned above for partly convective stars. Nevertheless, the lhs panel of Fig. 4 suggest that the observed magnetic field might not come from the tachocline but is the surface magnetic field generated by a distributed dynamo. The evolution of this large-scale field is governed by the strong field anchored in the tachocline and the stable layer underneath. The dynamo ingredients, naturally 
occurring in a global MHD simulation, will be presented in a forthcoming paper (Guerrero et al. 2017, in preparation).

\section{Discussion}

Global dynamo simulations have become an essential tool to understand the physics of the solar and stellar dynamos. The evolution of rotating turbulent convection of a plasma in hydrostatic equilibrium results in large-scale motions and magnetic fields. For the solar case, the models capture some of the observed characteristics of both, motions and magnetism. From the variety of global dynamo models in the literature, the ILES performed with the EULAG-MHD code have proven successful in reproducing two important features: the thin shear layer at the interface between the radiative and the convective zones, and the reversals of the large scale magnetic field in a time scale similar to the solar cycle. Based on the results of these models, in this proceeding we discussed the relevance of tachoclines for the generation of solar/stellar magnetic fields.

Three arguments are presented supporting the relevance of tachoclines. (i) the solar cycle period: according to estimations the value of the turbulent magnetic diffusivity in the bulk of the convection zone must be between $10^{8}-10^{9} \mathrm{~m}^{2} \mathrm{~s}^{-1}$. These value means a fast decay of the large-scale magnetic field and therefore a cycle period of about 2 years. In the global dynamo models the strong radial shear at the tachocline generates a strong toroidal magnetic field which keeps stored in the stable layer underneath. The co-existence of a shear profile together with a large-scale field is unstable and results in non-axisymmetric field and motions and therefore in a turbulence diffusivity even in the stable layer. This shear current instability develops obtaining energy from the magnetic field, when the magnetic field is not sufficiently enough, the instability decays. The time-scale of this balance sets the period of the oscillatory dynamo. (ii) The torsional oscillations: Guerrero et al. (2016b) reported a pattern of TO that resembles the observed pattern of speed-up and slow-down of the zonal flow observed in the solar surface. They demonstrated that in the simulation this pattern is formed in the tachocline due to the large-scale magnetic tension. The TO propagate upwards up to the surface of the model. The simulation also exhibit changes in the meridional flow pattern with the same period of the magnetic cycle. These changes are also observed at the solar surface. We found that the surface variation of the meridional circulation is a response to the TO, i.e., whenever the zonal flow speeds-up (slows-down), the residual of the meridional circulation is poleward (equatorward) such as it has been observed (Komm et al. 2015). (iii) Scaling of stellar magnetic fields with the Rossby number: the X-ray luminosity as well as the magnetic field show a well defined scaling with the Rossby number. For large values of Ro, buoyancy dominated convection, the magnetic field increases with the decrease of Ro; for smaller values of Ro, rotation dominated convection, there is a saturated regime. Global simulations of partly convective stars, with a solar-like stratification and in a wide range of Rossby numbers are able to reproduce a similar scaling profile with a $\mathrm{Ro}^{-1.79}$ for slow rotation rates and saturated for fast rotation rates. Models with radiative cores are necessary to reproduce this law. The observed magnetic field does not correspond to the magnetic field at the tacholine but to the surface magnetic field generated by a distributed dynamo. However, the large-scale field evolution is governed by the deep seated field anchored in the stable layer. On the other hand, solar-like models which consider only the convection zone do not reproduce the saturated regime but scale with $\mathrm{Ro}^{-1.2}$ in the buoyancy dominated regime. Further work is still necessary to determine if the same scaling law is reproduced in simulations of fully convective stars. Initial 
efforts have started in this sense in EULAG-MHD simulations (see Zaire et al., in this proceedings).

\section{Acknowledgements}

This work was partly funded by FAPEMIG grant APQ-01168/14 (GG and BZ), FAPESP grant 2013/10559-5 (EMGDP), CNPq grant 306598/2009-4 (EMGDP), NASA grants NNX09AJ85g and NNX14AB70G. PKS is supported by funding received from the European Research Council under the European Union's Seventh Framework Programme (FP7/2012/ERC Grant agreement no. 320375). The simulations were performed in the NASA cluster Pleiades and Brazilian super computer SDumont of the National Laboratory of Scientific Computation (LNCC).

\section{References}

Augustson, K., Brun, A. S., Miesch, M., \& Toomre, J. 2015, ApJ, 809, 149

Brandenburg, A. 2005, ApJ, 625, 539

Brown, B. P., Browning, M. K., Brun, A. S., Miesch, M. S., \& Toomre, J. 2008, ApJ, 689, 1354

Browning, M. K. 2008, ApJ, 676, 1262

Cally, P. S., Dikpati, M., \& Gilman, P. A. 2003, ApJ, 582, 1190

Cameron, R. H. \& Schüssler, M. 2016, A\& $A$, 591, A46

Chatterjee, P., Nandy, D., \& Choudhuri, A. R. 2004, A\&A, 427, 1019

Dikpati, M. \& Charbonneau, P. 1999, ApJ, 518, 508

Donati, J.-F. \& Brown, S. F. 1997, A\&GA, 326, 1135

Ghizaru, M., Charbonneau, P., \& Smolarkiewicz, P. K. 2010, ApJL, 715, L133

Guerrero, G. \& de Gouveia Dal Pino, E. M. 2008, A\& A, 485, 267

Guerrero, G., Dikpati, M., \& de Gouveia Dal Pino, E. M. 2009, ApJ, 701, 725

Guerrero, G. \& Käpylä, P. J. 2011, $A \& A$, 533, A40

Guerrero, G., Smolarkiewicz, P. K., de Gouveia Dal Pino, E. M., Kosovichev, A. G., \& Mansour, N. N. 2016a, ApJ, 819, 104

-. 2016b, ApJL, 828, L3

Guerrero, G., Zaire, B., Smolarkiewicz, P. K., et al. 2017

Käpylä, P. J., Mantere, M. J., \& Brandenburg, A. 2012, ApJL, 755, L22

Komm, R., González Hernández, I., Howe, R., \& Hill, F. 2015, Sol. Phys., 290, 3113

Landin, N. R., Mendes, L. T. S., \& Vaz, L. P. R. 2010, A\&A, 510, A46

Lawson, N., Strugarek, A., \& Charbonneau, P. 2015, ArXiv e-prints

Miesch, M. S., Gilman, P. A., \& Dikpati, M. 2007, ApJ, 168, 337

Miesch, M. S. \& Hindman, B. W. 2011, ApJ, 743, 79

Muñoz-Jaramillo, A., Nandy, D., \& Martens, P. C. H. 2011, ApJL, 727, L23

Parker, E. N. 1955, ApJ, 122, 293

Petit, P., Dintrans, B., Solanki, S. K., et al. 2008, MNRAS, 388, 80

Pizzolato, N., Maggio, A., Micela, G., Sciortino, S., \& Ventura, P. 2003, A\& A, 397, 147

Steenbeck, M., Krause, F., \& Rädler, K.-H. 1966, Zeitschrift Naturforschung Teil A, 21, 369

Vidotto, A. A., Gregory, S. G., Jardine, M., et al. 2014, MNRAS, 441, 2361

Wright, N. J. \& Drake, J. J. 2016, Nature, 535, 526

Wright, N. J., Drake, J. J., Mamajek, E. E., \& Henry, G. W. 2011, ApJ, 743, 48 